\title{
Drilling Processes Using Drag Bits and Its Application
}

\author{
Zhantao $\mathrm{Li}^{1}$ and Ken-ichi Itakura ${ }^{2}$ \\ 1. Ningbo University of Technology, Ningbo 315016, China \\ 2. Muroran Institute of Technology, Muroran 050-8585, Japan
}

\begin{abstract}
This paper proposes an analytical model to describe rock drilling processes using drag bits and rotary drills, and to induce relations among rock properties, bit shapes, and drilling parameters (rotary speed, thrust, torque, and stroke). In this model, a drilling process is divided into successive cycles. Each cycle includes two motions: feed and cutting. According to this model, drilling torque includes four components generated from cutting, friction, feed, and idle running respectively, the first three items are all proportional to the UCS (uniaxial compressive strength) when the penetration rate is constant. Laboratory tests verified the correctness and effectiveness of the proposed model qualitatively. Especially, the influence of friction on the flank face and the idle running was confirmed. Field experiments were performed. The results showed good correlation between the torque, penetration rate, and UCS. The proposed model and equations engender the possibility of eliminating useless components of cutting forces when investigating the relation between mechanical data and physical properties of rocks.
\end{abstract}

Key words: Rock cutting, rock drilling model, indentation, uniaxial compressive strength.

\section{Introduction}

During the last several decades, interest has been growing in the investigation of geophysical properties using drill performance parameters. Especially, in-situ estimation of UCS of rocks has been anticipated for use in mining and civil engineering fields because UCS is important for mining and tunnel design. However, most studies have used experimental methods to conclude experience equations describing relations among UCS and mechanical data. We believe that it is better to conduct research based on rock cutting theories.

In recent years, the emphasis related to rock cutting theories has shifted from analytical models to numerical models. Generally, numerical methods are useful to analyze and simulate cutting processes more precisely. However, an analytical model incorporates a group of equations to describe the relations among cutting forces, rock properties, bit shapes, and mechanical parameters. Therefore, research related to

Corresponding author: Ken-ichi Itakura, $\mathrm{PhD}$, professor, research fields: mining, rock mechanics, geotechnology. E-mail: itakura@mmm.muroran-it.ac.jp. analytical models remains important today.

Although many analytical models for rock cutting have been proposed, few analytical models are available for rock drilling. In this paper, the authors report a rock drilling model to describe rock drilling processes. This model consists of a series of successive cycles, each of which comprises a cutting motion and a penetration motion. Nishimatsu's analytical model [1] of rock cutting theories is used to study cutting motions, also, Mateus's formula [2] is used to analyze the cutting forces in feed operation. Finally, laboratory and field experiments are reported to demonstrate the usefulness of the proposed model for investigating relations between UCS and mechanical data.

\section{Proposal of an Analytical Model for Drag Bits}

\subsection{Drilling Model}

We propose a drilling analytical model as presented in Fig. 1. In this model, the rock is a cylinder: its wall thickness is equal to the blade length of the bit. A drilling process contains many drilling cycles, each of which consists of two steps: cutting and feed. Herein, 


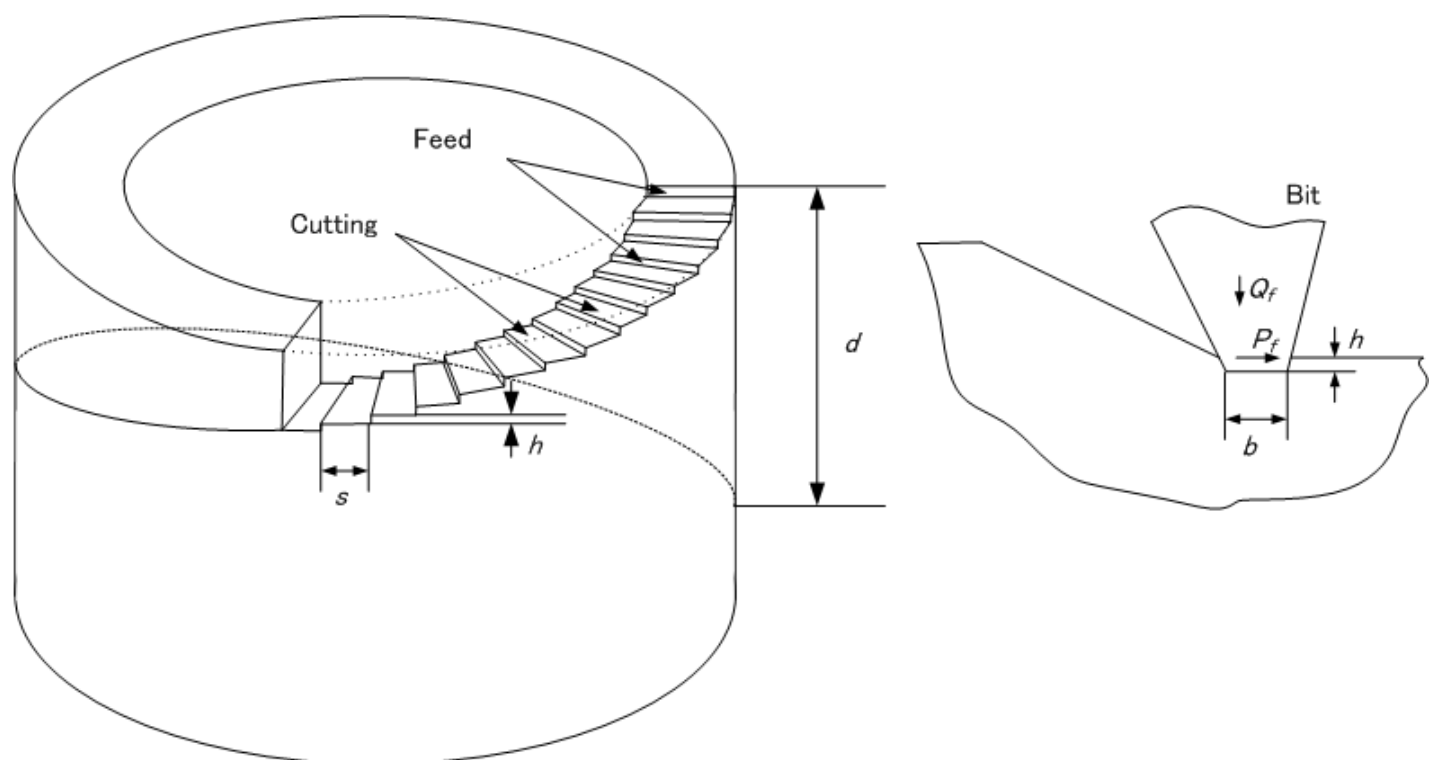

Fig. 1 Cutting and feed motions of a drag drill bit.

Nishimatsu's model [1] is expanded to analyze rock cutting motions in drilling. Feed is a complex operation when drilling. It might involve indentation, grinding, crushing, and smashing. However, for small-type drills, when the penetration rate per revolution is less than 0.5 $\mathrm{mm} / \mathrm{r}$, feed can be regarded approximately as an indentation. Herein, Mateus's formula [2] is expanded to analyze the cutting forces in feed operation.

\subsection{Cutting Motion}

Nishimatsu's theory is superior to other theories and models for showing cutting mechanisms. However, the bit wear is not considered in the model. Therefore, it cannot be used to analyze the effects of wear and friction on the flank face. In these analyses, we attempt to expand and enhance the Nishimatsu theory to fit actual cutting conditions.

Fig. 2 is the modified geometrical and mechanical model. Compared to the Nishimatsu model, a horizontal face is added on the flank face, it is designated as a virtual base. The virtual base area represents the degree of bit wear. The meanings of the signs in Fig. 2 are presented in Table 1.

For this study, the stress condition of the failure of rock is given by an envelope of Mohr's circle of stress. Therefore, we have

$$
\tau=\frac{1}{2} C_{0} \frac{1-\sin \phi_{i}}{\cos \phi_{i}}+\sigma \tan \phi_{i},
$$

where, $C_{0}$ is the rock's uniaxial compressive strength, and $\Phi_{i}$ is the angle of the rocks' internal friction.

According to Nishimatsu [1], the components of cutting force on the rake surface are

$$
P_{1}=\frac{1}{n+1} \cdot \frac{\left(1-\sin \phi_{i}\right) \sin \left(\beta+\phi_{j}\right)}{1+\cos \left(\beta+\phi_{i}+\phi_{j}\right)} \cdot d \cdot C_{0},
$$

where, $n$ is the stress distribution coefficient on the failure plane.

Considering the distribution condition of the stresses both on the rake surface and the virtual base, we can calculate the friction and normal forces on the virtual base as presented below.

$$
P_{2}=\frac{1}{n+1} \cdot \frac{\left(1-\sin \phi_{i}\right) \sin \beta \sin \phi_{j}}{1+\cos \left(\beta+\phi_{i}+\phi_{j}\right)} \cdot b \cdot C_{0} .
$$

In the equation, $b$ denotes the length of $\mathrm{a}_{1} \mathrm{a}_{5}$ in Fig. 2 (equivalent to the virtual base area).

\subsection{Feed Motion}

Mateus et al. [2] used a flat end indenter to perform the indentation tests. They concluded a relation between the indentation press and UCS, as presented below.

$$
p=k_{i} \cdot C_{0} \cdot d_{i}
$$

where, $p$ : indentation press $(\mathrm{Pa})$ 


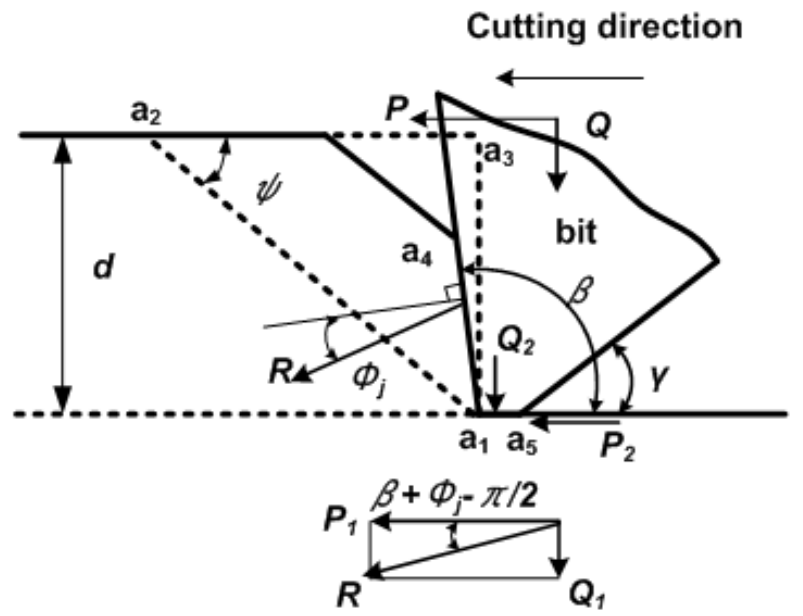

(a)

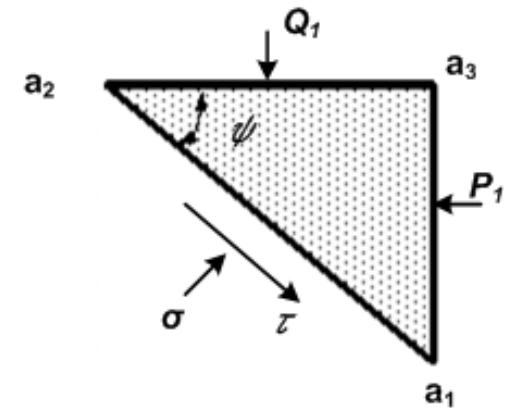

(b)

Fig. 2 Geometric and mechanical model for rock cutting.

Table 1 Meanings of the signs in Fig. 2.

\begin{tabular}{llll}
\hline Sign & Meaning & Sign & Meaning \\
\hline$\Phi_{j}$ & Friction angle between the bit and rock $R$ & $\begin{array}{l}\text { Cutting force per unit thickness } \\
\text { Main cutting force } R \text { (equivalent to machine torque if considering } \\
\beta\end{array}$ & $\begin{array}{l}\text { Blade angle of the bit } \\
\text { the distance from the axis) }\end{array}$ \\
$d$ & $\begin{array}{l}\text { Penetration per revolution } \\
\text { Angle between the free plane and failure } \\
\sigma, \tau\end{array}$ & $\begin{array}{l}\text { Back cutting force } R \text { (equivalent to thrust) } \\
\text { plane }\end{array}$ \\
$P_{l}, Q_{1}$ & \begin{tabular}{l} 
Components of the cutting force on the ${ }_{P_{2}, Q_{2}}$ rock surface \\
\hline
\end{tabular}
\end{tabular}

\section{$d_{i}$ : indentation depth (mm)}

$k_{i}$ : coefficient

We regard the bit in Fig. 1 as an indenter and evaluate the feed (indentation) force $Q_{f}$ using the following equation.

$$
Q_{f}=k_{i t} b \cdot d \cdot C_{0},
$$

Therein, $k_{i t}$ is a coefficient. Therefore the friction generated from indentation is

$$
P_{f}=Q_{f} \tan \phi_{j}=k_{i t} b \cdot d \cdot C_{0} \cdot \tan \phi_{j} .
$$

\subsection{Cutting Forces}

We analyzed the drilling process by regarding one drilling cycle as two steps for convenience: indentation and cutting. In fact, the two steps occur simultaneously in real drilling. The total torque can be written as shown below:

$$
T=k_{c} d \cdot C_{0}+k_{r} b \cdot C_{0}+k_{f} b \cdot d \cdot C_{0}+T_{i}
$$

In that equation, $k_{c}, k_{r}$, and $k_{f}$ are coefficients related to bit shapes and rock properties; $T_{i}$ denotes the torque when the machine is idle running. Eq. (7) shows that the total drilling torque includes four components: cutting torque, friction torque on the flank surface of the bit, feed (or indentation) torque, and idle running torque. The first item at the right is the torque caused by cutting, which is proportional to $d$, the penetration per revolution. The second item results from the friction on the flank face of the bit irrespective of the indentation process. The contact area of the flank surface (virtual base) can influence the friction force directly. The third item is the torque caused by feed motion (indentation), proportional to the product of $d$ and $b$. The fourth item $T_{i}$ is a constant for a drill.

\section{Laboratory/Field Experiments}

\subsection{Laboratory Experiments}

Several laboratory experiments were performed to verify the proposed model. Two-wing bits (outer 
diameter 28 mm, D4MB-1628-26, Sandvik Mining and Construction) were used in this research. Materials of four types were chosen: gypsum, cement, brick, and ore rock.

A new bit was used to avoid the influence of bit wear. The rotary speed was set in $580 \mathrm{r} / \mathrm{min}$. Drilling tests were conducted on materials of those four types. Mechanical data were recorded and analyzed. A Penetration Rate-Torque graph as shown in Fig. 3 was created to present the test results. Clearly, the changes of the slopes are explainable as the differences of UCS. The intercept of each line is the sum of the second item and fourth item in Eq. (7). The intercepts and slopes are presented in Table 2. They are all proportional to UCS. Therefore, the results verified the correctness of Eq. (7).

The third item in Eq. (7) contains the product of $b$ and $d$, which means that the wear of a bit can also engender some differences in the slopes, even for the same rocks and same bits. To investigate the effect of the bit wear, the blades of a bit were made flat-ended. The width of the reshaped flank surface was designated as $b$ (the virtual base length). Fig. 4 shows the different slopes when using the same bit but the wear degree of the bit blade was changed intentionally $(b=0.1,0.5$, $1.0 \mathrm{~mm})$. It confirmed the existence of the third item in Eq. (7).

\subsection{Field Experiments}

To investigate the relation between the drilling torque and UCS, several field experiments were performed at the roadway in G Coal Mine, Australia. A drilling system named Trussmaster mark II was used $[3,4]$. A part of the plan view of the site is presented in Fig. 5. In addition to two boreholes (55 mm diameter), 47 non-core holes (27 mm, $28 \mathrm{~mm}$, or $55 \mathrm{~mm}$ diameter, 5 m length) were drilled in the roof. Core 1 and No. 14 Hole nearby were taken as study objects.

Through the drilling process for No. 14 Hole, the drilling data-torque, thrust, rotational speed, and stroke-were measured and recorded. Then the data were analyzed and calculated. Good correlation was found between torque/penetration per revolution and UCS, as depicted in Fig. 6. Therefore, the results verified that Eq. (7) is correct using field experiment data.

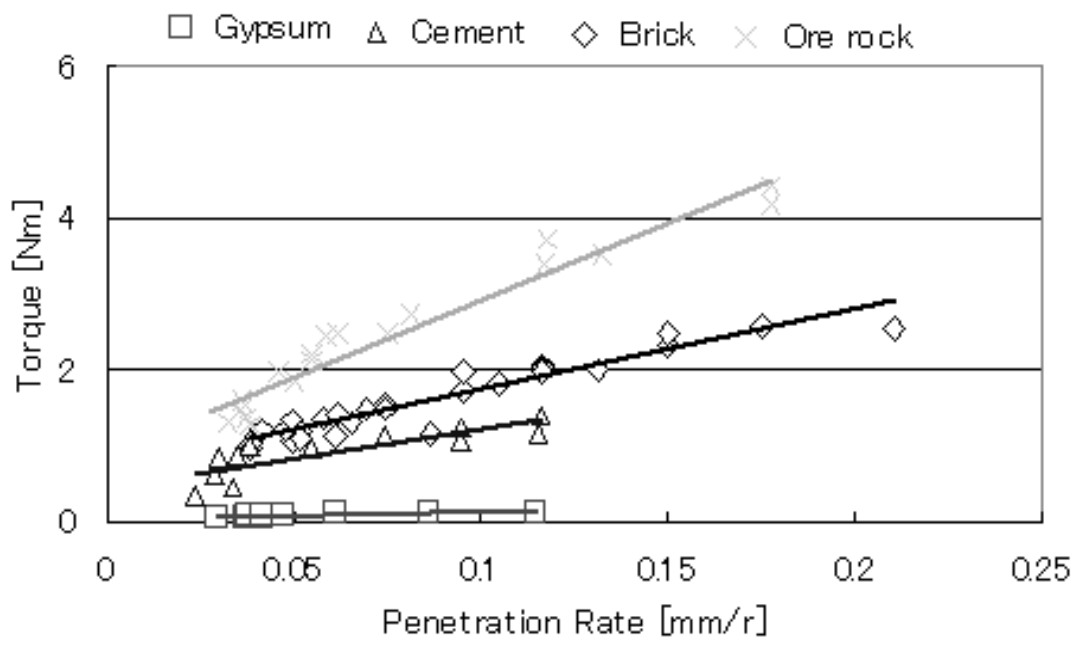

Fig. 3 Torque vs. Penetration Rate.

Table 2 Slopes and intercepts of the penetration rate - Torque lines.

\begin{tabular}{lccl}
\hline Material & UCS [MPa] & Slope & Intercept \\
\hline Gypsum & 0.3 & 0.9087 & 0.037 \\
Cement & 6.5 & 7.7979 & 0.444 \\
Brick & 12 & 10.803 & 0.6649 \\
Ore Rock & 20 & 20.398 & 0.906 \\
\hline
\end{tabular}




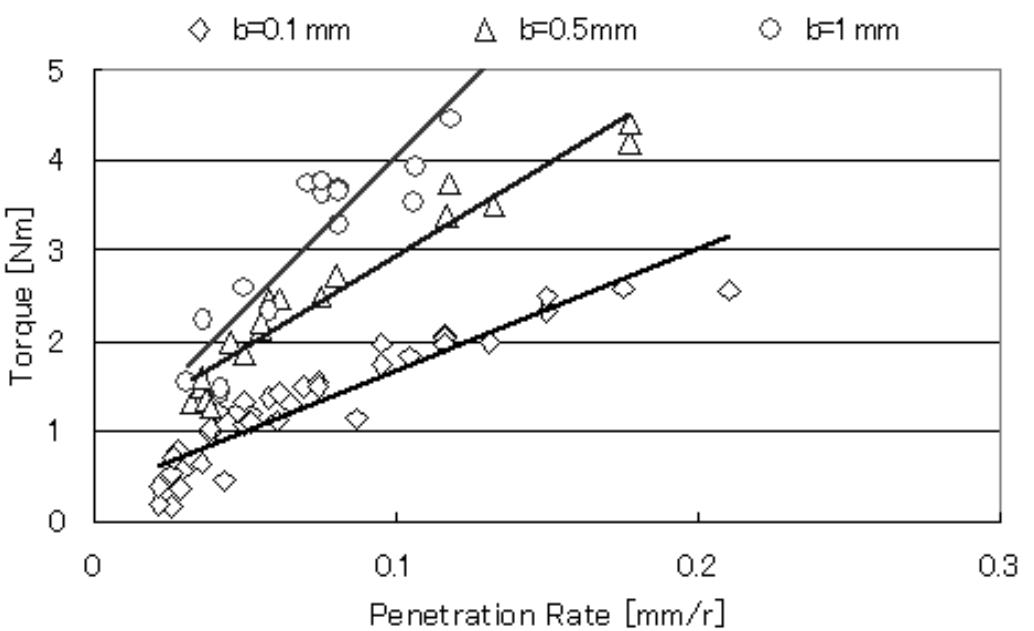

Fig. 4 Influence of the virtual base on drilling torque.

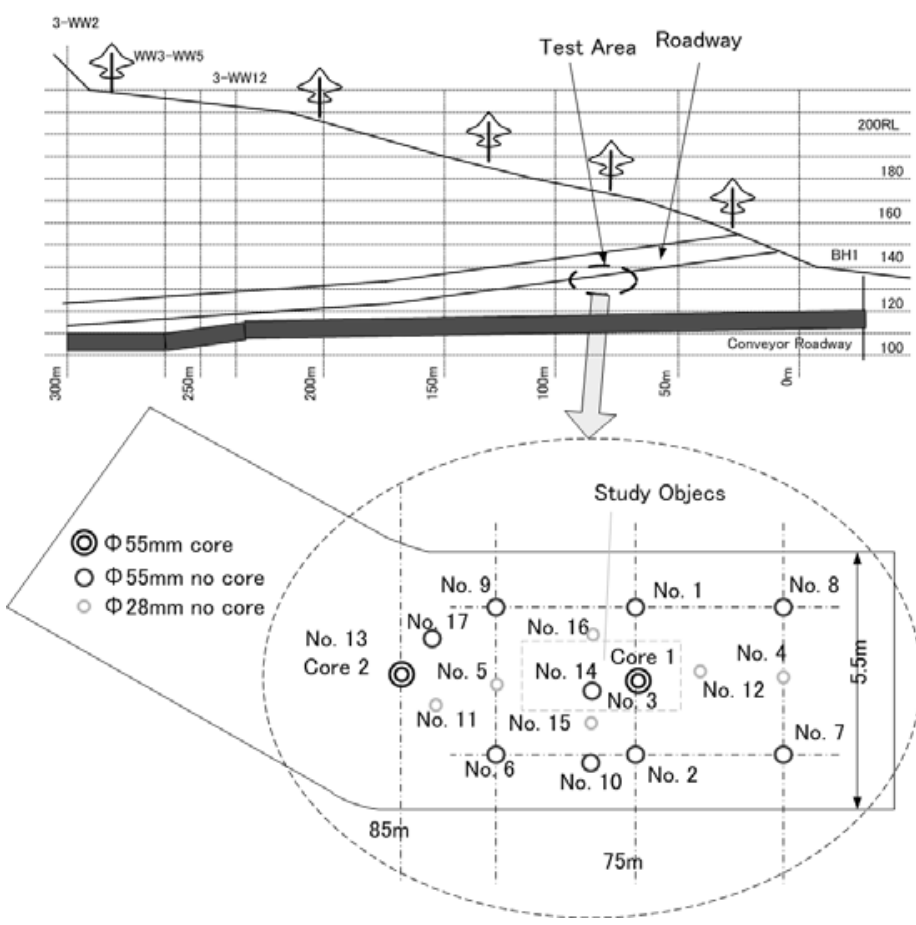

Fig. 5 Test site plan view of G Coal Mine.

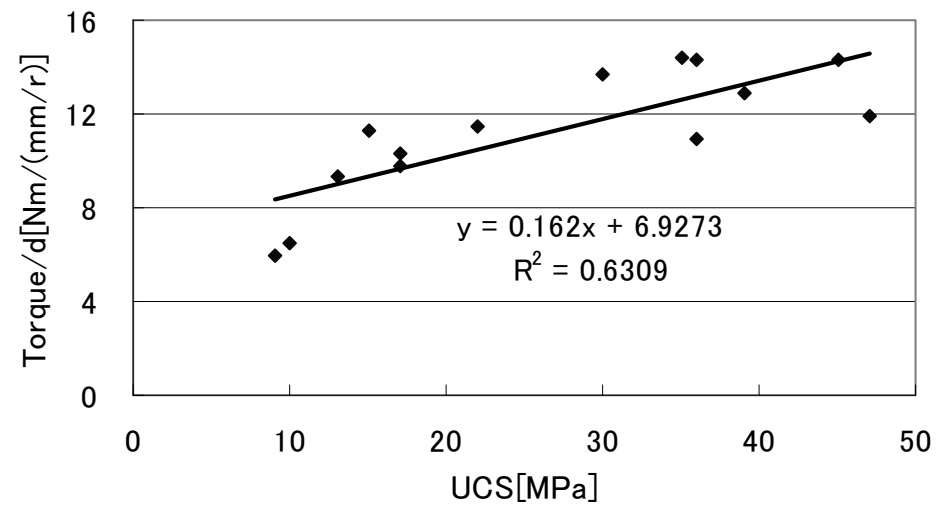

Fig. 6 Torque/d vs. UCS (G Coal Mine). 


\section{Conclusions}

An analytical model was proposed to describe drilling processes using drag bits. In this model, a drilling process is divided into successive cycles, each of which contains feed and cutting motions. Nishimatsu's rock cutting theory was expanded to analyze rock cutting with flat bits, and Mateus's indentation model is for feed motion.

Total drilling torque is resolved, respectively, into four components generated from cutting, friction on the flank surface, feed, and idle running. Cutting torque and feed torque, each of which is proportional to the penetration depth per revolution when UCS is constant, are the effective components of the total torque. Friction torque and idle running torque are not correlated to the penetration depth per revolution. Each is an ineffective component of the total torque.
Laboratory and field experiments were performed to verify the usefulness and correctness of the proposed model. Methods of predicting UCS based this model must be studied in future research.

\section{References}

[1] Y. Nishimatsu, The mechanics of rock cutting, International Journal of Rock Mechanics and Mining Sciences and Geomechanics Abstracts 9 (1972) 261-270.

[2] J. Mateus, N. F. Saavedra, Z. C. Carrillo and D. Mateus, Correlation development between indentation parameters and unconfined compressive strength for Colombian sandstones, Ciencia, Tecnología y Futuro 3 (3) (2007) 125-135.

[3] K. Itakura, T. Goto, Y. Yoshida, S. Tomita, S. Iguchi, Y. Ichihara and P. Mastalir, in: Aachen International Mining Symposium, Aachen, Germany, 2008, pp. 597-609.

[4] Z. Li, K. Itakura, S. Tomita, S. Iguchi, Y. Ichihara and P. Mastalir, in: 2009 Korea-Japan Joint Symposium on Rock Engineering, Suwon, Korea, 2009, pp. 57-65. 